Review

\title{
Vibrational Microspectroscopy for Cancer Screening
}

\section{Fiona M. Lyng ${ }^{1,2, *}$, Inês R. M. Ramos ${ }^{1,2}$, Ola Ibrahim ${ }^{1,2}$ and Hugh J. Byrne $^{3}$}

1 DIT Centre for Radiation and Environmental Science, FOCAS Research Institute, Dublin Institute of Technology, Kevin St, Dublin 8, Ireland; E-Mails: d11127684@mydit.ie (I.R.M.R.); ola.ibrahim@mydit.ie (O.I.)

2 School of Physics, Dublin Institute of Technology, Kevin St, Dublin 8, Ireland

3 FOCAS Research Institute, Dublin Institute of Technology, Kevin St, Dublin 8, Ireland; E-Mail: hugh.byrne@dit.ie

* Author to whom correspondence should be addressed; E-Mail: fiona.lyng@dit.ie; Tel.: +353-1-402-7972; Fax: +353-1-402-7901.

Academic Editor: Ken Ledingham

Received: 7 December 2014 / Accepted: 10 February 2015 / Published: 13 February 2015

\begin{abstract}
Vibrational spectroscopy analyses vibrations within a molecule and can be used to characterise a molecular structure. Raman spectroscopy is one of the vibrational spectroscopic techniques, in which incident radiation is used to induce vibrations in the molecules of a sample, and the scattered radiation may be used to characterise the sample in a rapid and non-destructive manner. Infrared (IR) spectroscopy is a complementary vibrational spectroscopic technique based on the absorption of IR radiation by the sample. Molecules absorb specific frequencies of the incident light which are characteristic of their structure. IR and Raman spectroscopy are sensitive to subtle biochemical changes occurring at the molecular level allowing spectral variations corresponding to disease onset to be detected. Over the past 15 years, there have been numerous reports demonstrating the potential of IR and Raman spectroscopy together with multivariate statistical analysis techniques for the detection of a variety of cancers including, breast, lung, brain, colon, oral, oesophageal, prostate and cervical cancer. This paper discusses the recent advances and the future perspectives in relation to cancer screening applications, focussing on cervical and oral cancer.
\end{abstract}

Keywords: optical diagnosis; lasers; Raman spectroscopy; FTIR spectroscopy; cervical cancer; oral cancer; screening; cytology 


\section{Introduction}

Cancer is a leading cause of disease worldwide with 14.1 million new cases, 8.2 million cancer deaths and 32.6 million people living with cancer (within five years of diagnosis) in 2012 [1]. Many cancers develop through pre-malignant stages which allow screening programmes to be implemented to detect cellular changes before invasive cancer develops. A good example is cervical cancer screening using the Papanicolaou (Pap) test which involves microscopic examination of cells exfoliated from the cervix.

Cytology is the study of disease at the cellular level and involves the preservation, staining and microscopic examination of cells that have been extracted from the body. Cytology can be used for practically any tissue type to detect abnormal cells. The principle of cytology is based on the fact that cancer cells lose their adhesive properties early in the disease process and can thus be easily exfoliated from tissue. An advantage of cytology is that sample collection is generally simple, rapid and painless.

Cytological screening aims to detect pre-cancer and cancer in a healthy population. In such populations, disease prevalence is relatively low and achieving a high test sensitivity is critical (see Figure 1). The aim of screening is to correctly identify as many cases of disease (positives) as possible while minimising incorrect identification of disease in individuals who do not have the disease (false positives) and negatives in individuals who do have the disease (false negatives). A false positive would result in a patient possibly undergoing unnecessary treatment while a false negative would result in a patient remaining undiagnosed and possibly progressing to cancer.

There is an unmet clinical need for new methods to aid cytologists in the early detection of pre-cancer. These methods ideally should be rapid, non-destructive, require minimal sample preparation and mostly importantly should not be subjective.

Sensitivity
- a measure of the ability of the
screening test to identify
positives
- expressed as the percentage of
positives correctly identified
by the test out of the total
number of positives actually
tested

Specificity
- a measure of the ability of the
screening test to identify
negatives
- expressed as the percentage of
negatives correctly identified
by the test out of the total
number of negatives actually
tested

Figure 1. Definition of sensitivity and specificity.

\section{Vibrational Spectroscopy}

Vibrational spectroscopy analyses vibrations within a molecule and the spectrum of vibrational energies or frequencies (in wavenumbers, $\mathrm{cm}^{-1}$ ) can be used to characterise a molecular structure. Every molecule has a unique spectrum which allows identification of the molecular compound and its abundance in a sample [2]. 
The exact energy required to excite a molecular vibration depends on the masses of the atoms involved in the vibration and the type of chemical bonds between these atoms and may be influenced by molecular structure, molecular interactions and the chemical microenvironment of the molecule.

Vibrational energies fall within the mid Infrared (IR) region of the electromagnetic spectrum and are commonly probed using IR absorption spectroscopy. IR spectroscopy is based on the absorption of infrared radiation by the sample and the fact that molecules absorb specific frequencies of the incident light which are characteristic of their structure.

A typical cellular FTIR spectrum is shown in Figure 2, together with a list of tentative spectral assignments in Table 1 [3]. The spectrum shown in Figure 2 can be divided into two regions, the high wavenumber region from $2500-4000 \mathrm{~cm}^{-1}$, corresponding to absorptions due to $\mathrm{O}-\mathrm{H}, \mathrm{N}-\mathrm{H}, \mathrm{C}-\mathrm{H}$ stretching vibrations, and the fingerprint region from $900-1800 \mathrm{~cm}^{-1}$, which consists of many overlapping bands (see Table 1), and is characteristic of the sample.

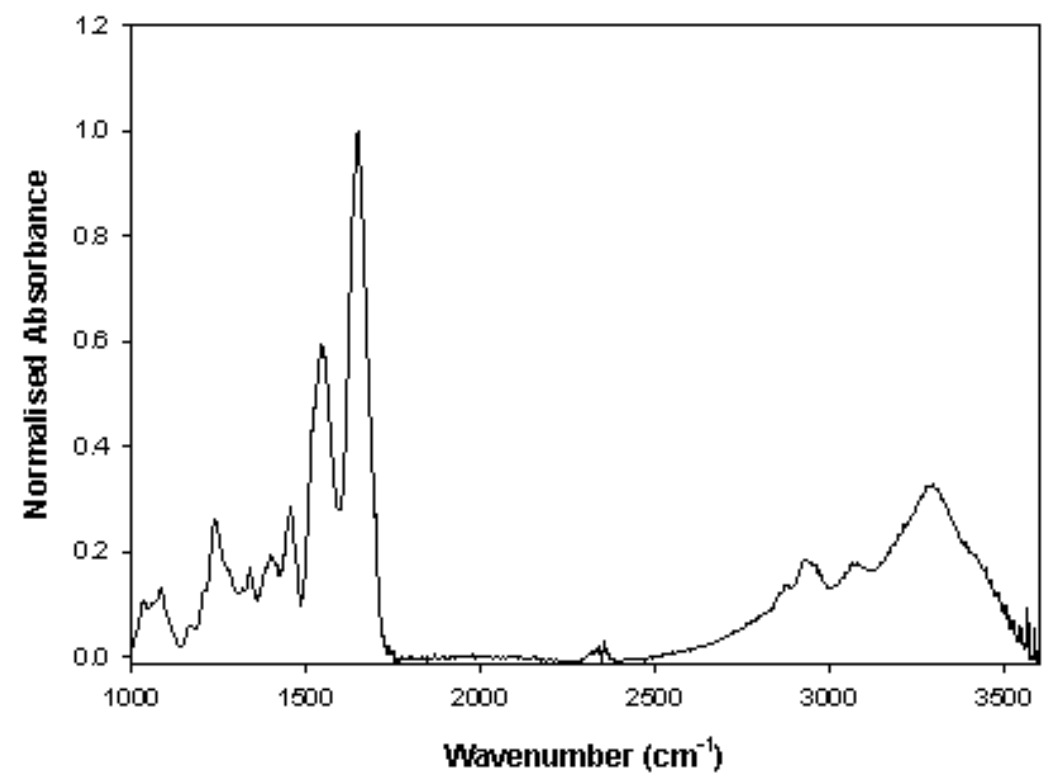

Figure 2. Typical FTIR spectrum from a population of cells.

Table 1. Tentative peak assignments [3] for typical FTIR cellular spectrum shown in Figure 2.

\begin{tabular}{cl}
\hline Wavenumber $\left(\mathbf{c m}^{-1}\right)$ & \multicolumn{1}{c}{ FTIR Peak Assignments } \\
\hline 1036 & $\mathrm{C}-\mathrm{C}$ skeletal stretching (proteins) \\
1080 & $\mathrm{PO}_{2}$ symmetric stretching (DNA/RNA) \\
1150 & $\mathrm{C}-\mathrm{C}$ and C-O stretching (proteins) \\
$1220-1280$ & $\mathrm{PO}_{2}$ asymmetric stretching (DNA/RNA), Amide III (C-N stretching, N-H bending, proteins) \\
1310 & $\mathrm{CH}_{2}$ stretching (phospholipids) \\
$1450-1470$ & $\mathrm{CH}_{3}$ asymmetric stretching (proteins, lipids) \\
$1520-1550$ & $\mathrm{Amide} \mathrm{II} \mathrm{(N-H} \mathrm{bending,} \mathrm{C-N} \mathrm{stretching,} \mathrm{proteins)}_{1620-1700} \mathrm{Amide} \mathrm{I} \mathrm{(C=O} \mathrm{stretching,} \mathrm{C-N} \mathrm{stretching} \mathrm{and} \mathrm{N-H} \mathrm{bending,} \mathrm{proteins)}_{2850}$ \\
2920 & $\mathrm{CH}_{2}$ symmetric stretching (lipids) \\
2960 & $\mathrm{CH}_{2}$ asymmetric stretching (lipids) \\
3290 & $\mathrm{NH}$ asymmetric stretching (lipids and proteins) \\
3400 & $\mathrm{OH}_{\text {stretching (water) }}$
\end{tabular}


Raman spectroscopy is an optical method based on inelastic light scattering. The sample is illuminated by monochromatic laser light and interactions between the incident photons and molecules in the sample result in scattering of the light. The coupling of the light generates vibrations within the material, which are again characteristic of the chemical structure, and the energy of the scattered light is reduced by an amount equal to the energy of the vibrational energy. Thus, the positions, relative intensities and shapes of the bands in a Raman spectrum carry detailed information about the molecular composition of the sample.

A typical cellular Raman spectrum is shown in Figure 3, together with a list of tentative spectral assignments in Table 2 [4]. As for Figure 2, the spectrum can be divided into two regions, the high wavenumber region, from $2500-3500 \mathrm{~cm}^{-1}$, and the fingerprint region, from $400-1800 \mathrm{~cm}^{-1}$.

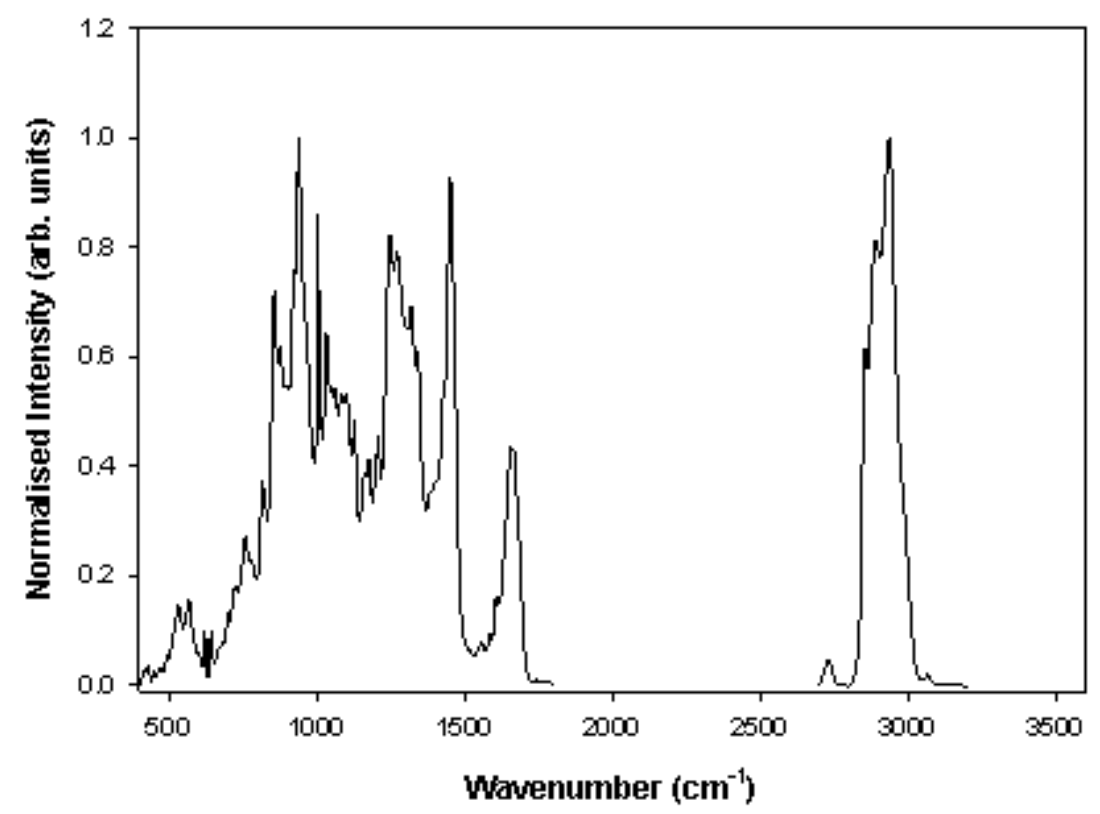

Figure 3. Typical cellular Raman spectrum.

Table 2. Tentative peak assignments [4] for typical Raman cellular spectrum shown in Figure 3.

\begin{tabular}{cl}
\hline Wavenumber $\left(\mathbf{c m}^{-1}\right)$ & \multicolumn{1}{c}{ Raman Peak Assignments } \\
\hline 621 & C-C twisting mode of Phenylalanine (proteins) \\
642 & C-C twisting mode of Tyrosine and Phenylalanine \\
670 & Thymine, Guanine (DNA/RNA) \\
720 & C-N stretching in Adenine and lipids \\
750 & symmetric breathing of Tryptophan (protein) \\
782 & Uracil, Thymine, Cytosine (ring breathing modes in the DNA/RNA) \\
827 & $\mathrm{PO}_{2}$ stretching in DNA, Tyrosine \\
854 & Ring breathing in Tyrosine and Proline (proteins) \\
935 & $\mathrm{C}-\mathrm{C}$ stretching mode of Proline and Valine \\
1003 & $\mathrm{C}-\mathrm{C}$ aromatic ring stretching in Phenylalanine \\
1030 & $\mathrm{C}-\mathrm{H}$ bending mode in Phenylalanine, C-N stretching in proteins \\
1080 & PO 2 symmetric stretching (DNA/RNA) \\
1085 & $\mathrm{C}-\mathrm{O}$ stretching \\
\hline
\end{tabular}


Table 2. Cont.

\begin{tabular}{cl}
\hline Wavenumber $\left(\mathbf{c m}^{-1}\right)$ & \multicolumn{1}{c}{ Raman Peak Assignments } \\
\hline 1130 & $\mathrm{C}-\mathrm{N}$ stretching in proteins; C-O stretching in carbohydrates \\
1155 & $\mathrm{C}-\mathrm{C}$ and $\mathrm{C}-\mathrm{N}$ stretching of proteins/lipids \\
1175 & $\mathrm{C}-\mathrm{H}$ in plane bending mode of Tyrosine and Phenylalanine; Cytosine, Guanine \\
1208 & $\mathrm{C}-\mathrm{C}_{6} \mathrm{H}_{5}$ stretching mode in Tryptophan, Phenylalanine \\
$1220-1280$ & Amide III (C-N stretching, N-H bending, proteins), $\mathrm{PO}_{2}$ asymmetric stretching (DNA/RNA) \\
1311 & $\mathrm{CH}_{3} / \mathrm{CH}_{2}$ twisting mode of collagen and lipid \\
1340 & Guanine (DNA/RNA), CH def. in proteins and carbohydrates \\
$1430-1460$ & $\mathrm{CH}\left(\mathrm{CH}_{2}\right)$ bending mode in proteins and lipids \\
1485 & Amide II (N-H bending, C-N stretching, proteins); Adenine, Guanine (DNA/RNA) \\
1580 & Adenine, Guanine (DNA/RNA); C=C bending mode of Phenylalanine \\
1615 & $\mathrm{C}=\mathrm{C}$ Phenylalanine, Tyrosine and Tryptophan \\
$1620-1700$ & Amide I (C=O stretching, C-N stretching and N-H bending, proteins) \\
2890 & $\mathrm{CH}_{2}$ symmetric stretching (lipids) \\
2940 & $\mathrm{CH}_{2}$ and $\mathrm{CH}_{3}$ symmetric stretching (lipids) \\
2980 & $\mathrm{CH}_{3}$ symmetric stretching (lipids) \\
\hline
\end{tabular}

Raman and IR spectroscopy are complementary techniques. IR spectroscopy involves the absorption of radiation whereas Raman spectroscopy is a scattering technique, whereby the incident radiation couples with the vibrating polarisation of the molecule and generates or annihilates a vibration. These different underlying mechanisms give rise to the complementarity of the two techniques and they each have different selection rules. For a vibration to be active in IR spectroscopy, a change in dipole is required, whereas a change in polarisability is required for a vibration to be Raman active. Vibrations of asymmetric, polar bonds tend to be strong in IR spectra, whereas symmetric, nonpolar groups are strong in Raman spectra. For example, O-H vibrations of water are very strong in IR spectra, whereas they are relatively weak in Raman spectra. This feature means that Raman spectroscopy may be a more suitable technique for biological applications. Another difference is that Raman spectroscopy can be employed in the UV, visible or near IR regions of the electromagnetic spectrum whereas IR spectroscopy monitors the absorption of IR radiation. Therefore, Raman spectroscopy has a higher spatial resolution ( $<1 \mu \mathrm{m}$ for Raman, $\sim 5-10 \mu \mathrm{m}$ for IR). Raman spectroscopy is, however, inherently weak with only a small percentage of photons undergoing Raman scattering $\left(\sim 10^{-6}\right)$. Longer spectral acquisition times are therefore needed to achieve adequate signal to noise in Raman compared to IR spectroscopy.

IR and Raman spectra of biomedical samples such as cells and tissues are a superposition of all the contributions from each individual biochemical component such as DNA, RNA, proteins, lipids and carbohydrates. Thus vibrational spectroscopy methods can provide a molecular diagnosis by providing a "biochemical fingerprint" of the complete genome, proteome and metabolome of the cell or tissue [5]. This can be provided in a rapid, label free, non-destructive manner allowing additional analyses to be performed subsequently, such as staining, immunocytochemistry etc. Standardised methods and procedures for collecting IR spectra and images from biological samples have recently been described [6].

In recent years, Raman and FTIR spectroscopy have been used in the detection of a variety of cancers, including breast, prostate, oesophageal, colon, lung, oral and cervical cancer, and excellent sensitivity and specificity values have been reported [7-9]. 
For the purposes of this review, cervical and oral cancers will be focussed on as these tissues are readily accessible and amenable to cytological screening.

\section{Cervical Cancer}

Cervical cancer is the fourth most common cancer in women worldwide with an estimated 528,000 new cases and 266,000 deaths in 2012 [1]. The mortality associated with cervical cancer can be reduced if this disease is detected at the early stages of development or at the pre-malignant state (cervical intraepithelial neoplasia, CIN).

Currently, a Papanicolaou test is used to screen for CIN and cervical cancer in the general female population. The Papanicolaou test (also called Pap smear, Pap test, cervical smear, or smear test) is a screening test used to detect cancer and pre-cancer in the ectocervix. A spatula or cytobrush is used to collect the cells from the cervix and after placing in liquid fixative, the cells are then collected on a filter and transferred to a slide and stained. The cells are examined for abnormalities under the microscope by highly trained personnel. The Pap test is an effective, widely used method for early detection of cervical cancer and pre-cancer. Despite a high specificity of $95 \%-98 \%$, the sensitivity of the Pap test is reported to vary greatly from $74 \%$ to $96 \%$ [10]. Low grade dysplasia or CIN 1 can spontaneously regress without leading to cervical cancer. However some CIN 1 lesions progress to moderate (CIN 2) and subsequently severe dysplasia (CIN 3), finally progressing to invasive cancer [11]. An abnormal Pap smear is followed by colposcopy, biopsy and histological confirmation of the diagnosis. This involves the visual examination of histological sections by highly trained personnel. The grading characteristics are quite subjective and pre-malignancy may not be visually perceptible at all.

Persistent infection with high-risk human papillomavirus (HPV) (e.g., HPV types 16, 18) is accepted as the major cause for the development of cervical pre-cancer and cancer [12]. HPV testing has recently been introduced in parallel with Pap smear screening for safe and cost-effective lengthening of the cervical screening interval due to its high-negative predictive values [13]. The HPV DNA test identifies high-risk HPV oncogene expression. However, this test is expensive, time-consuming and requires experienced technicians. Over the last decade, prophylactic HPV vaccines have been developed but despite the introduction of these vaccines, there is still a need for regular cervical screening, as the vaccines do not protect against all oncogenic HPV types. Additionally, some women may not benefit from the vaccines if there is a pre-existing HPV infection or they do not receive the complete number of doses (three doses over a 6-month period). After vaccination, women must still get routine Pap tests which can detect abnormal cervical growth regardless of what HPV type causes it to develop.

The first investigations of cervical exfoliated cells using IR spectroscopy were in the early 1990's by Wong and co-workers. They published a number of papers showing significant differences between normal, pre-cancer and cancer samples [14-17].

These initial studies recorded spectra from cell pellets rather than from individual cells and a number of confounding factors such as polymorphs, endocervical columnar cells, metaplastic cells, cervical mucus and debris were subsequently identified [18]. Further investigations by other groups identified similar confounding factors such as inflammation, metaplasia, hormonal changes, metabolic activity, blood, mucus [19-23]. 
However, an important early study by Cohenford and Rigas [24] showed that the FTIR spectra of morphologically normal exfoliated cells from women with dysplasia or cancer exhibit extensive IR spectroscopic changes. This finding was later confirmed by Schubert et al. [25] who showed spectral changes in cytologically normal cells, most likely due to HPV infection.

Walsh et al. [26] employed attenuated total reflection (ATR) FTIR spectroscopy to show spectral differences between normal and low grade and high grade dysplasia cytology samples. ATR-FTIR spectroscopy involves the IR beam being directed through a high refractive index crystal, typically diamond, germanium or zinc, where it undergoes total internal reflection generating an evanescent waveform that interacts with the biological sample at the surface of the crystal. Interestingly, a recent ATR-FTIR study has shown that cervical pre-cancer is more accurately identified when histology rather than cytology is used as the gold standard to classify the samples [27].

ATR-FTIR spectroscopy has also been shown to be capable of distinguishing between high risk HPV infections in women with low grade and high grade dysplasia [28]. Interestingly, spectral differences were also found between women in their 20 s and in their 30 s in samples of the same cytological grade and HPV type, most likely because of a persistent HPV infection. Schubert et al. [25] also showed that FTIR spectroscopy could discriminate cervical exfoliated cells based on their HPV status. In addition, a number of studies have used cervical cell lines as model systems to show the ability of vibrational spectroscopy (both IR and Raman) to differentiate cells based on HPV status [28-33].

In contrast to the extensive work on exfoliated cervical cells using IR spectroscopy, there are relatively few studies using Raman spectroscopy, most likely because of the issues with confounding factors.

Vargis et al. [33] showed that Raman spectroscopy could classify HPV-positive and -negative cytology samples with an accuracy of $98.5 \%$. A study by Rubina et al. [34] used Raman spectroscopy to distinguish between normal and cervical cancer cytology samples. As the spectra of cervical cancer samples were dominated by blood features, cytology samples were treated with red blood cell lysis buffer prior to Raman acquisition. A classification accuracy of $\sim 80 \%$ was obtained. Both of these studies used cell pellets rather than recording Raman spectra from individual cells and this probably resulted in the relatively low classification accuracy in the study by Rubina et al. due to sample heterogeneity.

A recent study by Bonnier et al. [35] addressed many of the issues involved in recording Raman spectra from ThinPrep cervical cytology samples. A new method based on pre-treatment of the slides with hydrogen peroxide to clear blood residue contamination before Raman recording was shown to minimise variability within the data sets. This resulted in the collection of highly reproducible data with clear discrimination between negative cytology and CIN cytology. Importantly, all data was recorded on glass ThinPrep slides which are currently used for clinical cervical cytology rather than spectroscopic substrates such as MirrIR or calcium fluoride substrates which are commonly used for research purposes and, although they reduce the presence of confounding contributions of the substrate, are considerably more expensive.

\section{Oral Cancer}

Oral cancer is defined as squamous cell carcinoma of the lip, oral cavity and oropharynx, and is the sixth most common malignancy in the world [36]. Patients with oral squamous cell carcinoma (OSCC) often have a poor outcome even though advances in treatment have been made. The 5 year survival 
rate is approximately $80 \%$ for early stage disease but only approximately $20 \%$ for late stage disease [37] and patients often present with advanced disease. Multiple primary tumours are common and second primary tumours are the most common cause of death in patients with early stage disease [38].

OSCC is a multi causal disease and risk factors include lifestyle habits (tobacco exposure and alcohol consumption), dietary factors, occupational activity, socioeconomic status, exposure to external agents, and genetic susceptibility $[39,40]$. Most cases of oral cancer occur in patients of 60 years or over and the risk increases with advancing age.

The gold standard method for diagnosis of oral cancer and pre-malignant lesions is clinical examination and histopathological evaluation of biopsied tissue [41]. Conventional visual and tactile examination (CVTE) is used to detect OSCC and pre-malignant oral lesions in a screening setting but oral pre-malignant lesions and some early OSCC can be difficult to distinguish from inflammatory lesions. Most lesions are white patches known as leukoplakia and only approx. $4 \%$ of these lesions progress to cancer. CVTE cannot distinguish between these lesions and non-progressive lesions. Biopsy is followed by histopathology, where the excised tissue is processed, cut into sections and mounted on a glass slide for examination by a pathologist. Grading of tumours depends on the visual assessment of tissue architecture and characteristics of individual cells. The grading characteristics used are highly subjective and early proliferation/pre-malignancy may not be apparent morphologically. In addition, it can be difficult to discriminate between reactive/inflammatory lesions and pre-cancer lesions.

A number of screening and diagnostic aids for early detection and diagnosis of oral cancer and pre-cancer have become available over the last decade including brush biopsy, toluidine blue vital staining, post-acetic acid rinse tissue reflectance and tissue autofluorescence. Some of these techniques show promise but as yet none has been definitively proven to perform better than conventional visual examination [42]. Well designed clinical studies are needed where the techniques are directly compared to the diagnostic gold standard, scalpel biopsy and histopathology.

Early detection of cancer greatly increases the chances for successful treatment. There is a significant clinical need for new techniques that can detect pre-malignant changes that may progress to cancer. Oral cancer progression is not as clear cut as, for example, in cervical cancer in which there is a well-defined progression from mild to moderate to severe dysplasia (CIN 1, 2 and 3). The majority of histologically pre-malignant oral lesions do not progress to cancer at all and mild dysplasia can progress to cancer as readily as severe dysplasia. In a long term follow up of 236 patients with oral lesions, Holmstrup et al. [43] found that the degree of dysplasia was not a predictor of malignant potential of the lesions. The inability of conventional histopathology to predict which lesions will progress to cancer underlines the need for new techniques or new technologies to address this clinical need.

While a number of Raman spectroscopy studies have been carried out on oral tissues [44-46], to date, only a limited number of IR studies have focused on oral exfoliated cells.

Papamarkakis et al. [47] showed that oral cells could be classified according to anatomical region with cells from cheek, palate and gums being spectrally similar whereas cells from the tongue and from the floor of the mouth were spectrally distinct due to an abundance of keratin and collagen respectively. It was further shown that samples from patients with reactive atypical changes or malignancy associated changes were more spectrally similar to a sample from a patient with squamous cell carcinoma than to samples from healthy volunteers [47]. A further IR study from the same group [5,48] showed that spectra from squamous cells collected from the tongues of healthy volunteers could be discriminated 
from squamous cells collected from the tongues of patients with oral disease, mostly dysplastic and cancer cases, using unsupervised principal components analysis. An artificial neural network was trained to automatically distinguish the clinical cases from the normal cases and sensitivity and specificity values of $96 \%$ and $94.3 \%$ were obtained. Interestingly, these results were obtained from morphologically normal cells, the majority of cells present in exfoliated cell samples. This indicates that biochemical changes can be detected by IR spectroscopy before morphological changes are apparent.

\section{Future Perspectives}

Vibrational spectroscopy shows great potential as a label free, high sensitivity and specificity test for cervical and oral cancer screening. The respective techniques of Raman and IR spectroscopy each has its own potential advantages for clinical applications (reviewed in [49]), with Raman spectroscopy having a higher spatial resolution, minimal sample preparation, and minimal interference from water and FTIR spectroscopy having a higher signal to noise ratio allowing faster acquisition times. ATR-FTIR spectroscopy can achieve higher spatial resolution and can allow measurement from aqueous samples. Recent advances in these techniques have included surface enhanced Raman spectroscopy (SERS), which allows significant enhancement of the Raman signal through close proximity/adsorption onto a roughened metal surface, a colloidal solution or a roughened electrode, tip enhanced Raman spectroscopy (TERS) which combines the high spatial resolution of atomic force microscopy with SERS, coherent anti-Stokes Raman scattering (CARS) and stimulated Raman scattering (SRS) (reviewed in [50]) which allow rapid real time imaging of single cells, and the development of high brightness quantum cascade lasers to improve acquisition times for IR imaging. For cytology applications, a key consideration is that these spectroscopic techniques could fit into the current workflow. Both Raman and IR spectroscopy can be operated in microscopic mode and, with automated spectral acquisition, these techniques could be similar to the Thinprep Imager (Hologic) or the Focal Point GS Imaging system (BD), which use image processing algorithms to automatically review liquid based cytology Thinprep and SurePath slides. Both Raman and IR spectroscopy are reagent free, so costs should be favourable when compared to the imaging systems or to manual scoring where personnel costs could be reduced. A recent study [35] has shown that glass ThinPrep slides can be used for Raman spectral recording whereas spectroscopic substrates, such as MirrIR or calcium fluoride substrates, are used for IR spectroscopy and although they reduce the presence of confounding contributions of the substrate, they are considerably more expensive than the glass slides used in the cytology laboratory.

However, much work still remains before these techniques could be translated into standard clinical practice [51]. Many studies have used relatively small sample sizes and as such may be biased. Screening populations are heavily weighted towards negative results with only a small percentage of positive results so validation in large multi centre studies is needed using real world cytology samples from screening populations. Large scale clinical trials are needed to obtain the large volumes of data necessary for the development of robust classification algorithms and histopathology rather than cytology should be used to classify the samples.

If spectroscopy can be shown to be as good as, or better than, the gold standard histopathology, then there is great potential for these techniques to be used as an alternative or an adjunct to the current cytology screening methods. The advantages would be higher accuracy, higher throughput 
and reduced workload for the cytologist/pathologist and higher accuracy and chance of earlier detection for the patient.

\section{Acknowledgments}

The authors acknowledge funding from Enterprise Ireland co-funded by the European Regional Development Fund (ERDF) and Ireland's EU Structural Funds Programme 2007-2013, CF2011 1045, Science Foundation Ireland, 12/IP/1494, and Dublin Institute of Technology Fiosraigh Research Excellence Award.

\section{Author Contributions}

F.M.L. conceived the idea for the manuscript and wrote the manuscript with input from I.R., O.I. and H.J.B.

\section{Conflicts of Interest}

The authors declare no conflict of interest.

\section{References}

1. Ferlay, J.; Soerjomataram, I.; Ervik, M.; Dikshit, R.; Eser, S.; Mathers, C.; Rebelo, M.; Parkin, D.M.; Forman, D.; Bray, F. GLOBOCAN, 2012 v1.0, Cancer Incidence and Mortality Worldwide: IARC CancerBase No. 11, 2013. International Agency for Research on Cancer Web site. Available online: http://globocan.iarc.fr (accessed on 24 November 2014).

2. Diem, M. Introduction to Modern Vibrational Spectroscopy; Wiley: New York, NY, USA, 1993.

3. Movasaghi, Z.; Rehman, S.; Rehman, I.U. Fourier Transform Infrared (FTIR) Spectroscopy of Biological Tissues. Appl. Spectrosc. Rev. 2008, 43, 134-179.

4. Movasaghi, Z.; Rehman, S.; Rehman, I.U. Raman Spectroscopy of Biological Tissues. Appl. Spectrosc. Rev. 2007, 42, 493-541.

5. Diem, M.; Mazur, A.; Lenau, K.; Schubert, J.; Bird, B.; Miljkovic, M.; Krafft, C.; Popp, J. Molecular pathology via IR and Raman spectral imaging. J. Biophotonics 2013, 6, 855-886.

6. Baker, M.J.; Trevisan, J.; Bassan, P.; Bhargava, R.; Butler, H.J.; Dorling, K.M.; Fielden, P.R.; Fogarty, S.W.; Fullwood, N.J.; Heys, K.A.; et al. Using Fourier transform IR spectroscopy to analyze biological materials. Nat. Protoc. 2014, 9, 1771-1791.

7. Ellis, D.I.; Cowcher, D.P.; Ashton, L.; O’Hagan, S.; Goodacre, R. Illuminating disease and enlightening biomedicine: Raman spectroscopy as a diagnostic tool. Analyst 2013, 138, 3871-3884.

8. Kendall, C.; Isabelle, M.; Bazant-Hegemark, F.; Hutchings, J.; Orr, L.; Babrah, J.; Baker, R.; Stone, N. Vibrational spectroscopy: A clinical tool for cancer diagnostics. Analyst 2009, 134, 1029-1045.

9. Nijssen, A.; Koljenovic, S.; Bakker Schut, T.C.; Caspers, P.J.; Puppels, G.J. Towards oncological application of Raman spectroscopy. J. Biophotonics 2009, 2, 29-36. 
10. Kitchener, H.C.; Blanks, R.; Cubie, H.; Desai, M.; Dunn, G.; Legood, R.; Gray, A.; Sadique, Z.; Moss, S.; MAVARIC Trial Study Group. MAVARIC - A comparison of automation-assisted and manual cervical screening: A randomised controlled trial. Health Technol. Assess. 2011, 15, iii-iv, ix-xi, 1-170.

11. Koss, L.G.; Melamed, M.R. Koss' Diagnostic Cytology and Its Histopathologic Bases; Lippincott Williams \& Wilkins: Philadelphia, PA, USA, 2006.

12. Walboomers, J.M.; Jacobs, M.V.; Manos, M.M.; Bosch, F.X.; Kummer, J.A.; Shah, K.V.; Snijders, P.J.; Peto, J.; Meijer, C.J.; Munoz, N. Human papillomavirus is a necessary cause of invasive cervical cancer worldwide. J. Pathol. 1999, 189, 12-19.

13. Schiffman, M.; Wentzensen, N.; Wacholder, S.; Kinney, W.; Gage, J.C.; Castle, P.E. Human papillomavirus testing in the prevention of cervical cancer. J. Natl. Cancer Inst. 2011, 103, 368-383.

14. Fung Kee Fung, M.; Senterman, M.; Eid, P.; Faught, W.; Mikhael, N.Z.; Wong, P.T. Comparison of Fourier-transform infrared spectroscopic screening of exfoliated cervical cells with standard Papanicolaou screening. Gynecol. Oncol. 1997, 66, 10-15.

15. Neviliappan, S.; Fang Kan, L.; Tiang Lee Walter, T.; Arulkumaran, S.; Wong, P.T.T. Infrared spectral features of exfoliated cervical cells, cervical adenocarcinoma tissue, and an adenocarcinoma cell line (SiSo). Gynecol. Oncol. 2002, 85, 170-174.

16. Wong, P.T.; Wong, R.K.; Caputo, T.A.; Godwin, T.A.; Rigas, B. Infrared spectroscopy of exfoliated human cervical cells: Evidence of extensive structural changes during carcinogenesis. Proc. Natl. Acad. Sci. USA 1991, 88, 10988-10992.

17. Yazdi, H.M.; Bertrand, M.A.; Wong, P.T. Detecting structural changes at the molecular level with Fourier transform infrared spectroscopy. A potential tool for prescreening preinvasive lesions of the cervix. Acta Cytol. 1996, 40, 664-668.

18. Wong, P.T.T.; Senterman, M.K.; Jackli, P.; Wong, R.K.; Salib, S.; Campbell, C.E.; Feigel, R.; Faught, W.; Fung Kee Fung, M. Detailed account of confounding factors in interpretation of FTIR spectra of exfoliated cervical cells. Biopolymers 2002, 67, 376-386.

19. Chiriboga, L.; Xie, P.; Vigorita, V.; Zarou, D.; Zakim, D.; Diem, M. Infrared spectroscopy of human tissue. II. A comparative study of spectra of biopsies of cervical squamous epithelium and of exfoliated cervical cells. Biospectroscopy 1998, 4, 55-59.

20. Cohenford, M.A.; Godwin, T.A.; Cahn, F.; Bhandare, P.; Caputo, T.A.; Rigas, B. Infrared spectroscopy of normal and abnormal cervical smears: Evaluation by principal component analysis. Gynecol. Oncol. 1997, 66, 59-65.

21. Diem, M.; Chiriboga, L.; Lasch, P.; Pacifico, A. IR spectra and IR spectral maps of individual normal and cancerous cells. Biopolymers 2002, 67, 349-353.

22. Romeo, M.J.; Quinn, M.A.; Burden, F.R.; McNaughton, D. Influence of benign cellular changes in diagnosis of cervical cancer using IR microspectroscopy. Biopolymers 2002, 67, 362-366.

23. Wood, B.R.; Quinn, M.A.; Tait, B.; Ashdown, M.; Hislop, T.; Romeo, M.; McNaughton, D. FTIR microspectroscopic study of cell types and potential confounding variables in screening for cervical malignancies. Biospectroscopy 1998, 4, 75-91.

24. Cohenford, M.A.; Rigas, B. Cytologically normal cells from neoplastic cervical samples display extensive structural abnormalities on IR spectroscopy: Implications for tumor biology. Proc. Natl. Acad. Sci. USA 1998, 95, 15327-15332. 
25. Schubert, J.M.; Bird, B.; Papamarkakis, K.; Miljkovic, M.; Bedrossian, K.; Laver, N.; Diem, M. Spectral cytopathology of cervical samples: Detecting cellular abnormalities in cytologically normal cells. Lab. Invest. 2010, 90, 1068-1077.

26. Walsh, M.J.; Singh, M.N.; Pollock, H.M.; Cooper, L.J.; German, M.J.; Stringfellow, H.F.; Fullwood, N.J.; Paraskevaidis, E.; Martin-Hirsch, P.L.; Martin, F.L. ATR microspectroscopy with multivariate analysis segregates grades of exfoliative cervical cytology. Biochem. Biophys. Res. Commun. 2007, 352, 213-219.

27. Gajjar, K.; Ahmadzai, A.A.; Valasoulis, G.; Trevisan, J.; Founta, C.; Nasioutziki, M.; Loufopoulos, A.; Kyrgiou, M.; Stasinou, S.M.; Karakitsos, P.; et al. Histology verification demonstrates that biospectroscopy analysis of cervical cytology identifies underlying disease more accurately than conventional screening: Removing the confounder of discordance. PLoS One 2014, 9, e82416.

28. Kelly, J.G.; Cheung, K.T.; Martin, C.; O’Leary, J.J.; Prendiville, W.; Martin-Hirsch, P.L.; Martin, F.L. A spectral phenotype of oncogenic human papillomavirus-infected exfoliative cervical cytology distinguishes women based on age. Clin. Chim. Acta 2010, 411, 1027-1033.

29. Jess, P.R.T.; Smith, D.D.W.; Mazilu, M.; Dholakia, K.; Riches, A.C.; Herrington, C.S. Early detection of cervical neoplasia by Raman spectroscopy. Int. J. Cancer 2007, 121, 2723-2728.

30. Kim, D.-H.; Jarvis, R.M.; Allwood, J.W.; Batman, G.; Moore, R.E.; Marsden-Edwards, E.; Hampson, L.; Hampson, I.N.; Goodacre, R. Raman chemical mapping reveals site of action of HIV protease inhibitors in HPV16 E6 expressing cervical carcinoma cells. Anal. Bioanal. Chem. 2010, 398, 3051-3061.

31. Ostrowska, K.M.; Garcia, A.; Meade, A.D.; Malkin, A.; Okewumi, I.; O’Leary, J.J.; Martin, C.; Byrne, H.J.; Lyng, F.M. Correlation of p16(INK4a) expression and HPV copy number with cellular FTIR spectroscopic signatures of cervical cancer cells. Analyst 2011, 136, 1365-1373.

32. Ostrowska, K.M.; Malkin, A.; Meade, A.; O’Leary, J.; Martin, C.; Spillane, C.; Byrne, H.J.; Lyng, F.M. Investigation of the influence of high-risk human papillomavirus on the biochemical composition of cervical cancer cells using vibrational spectroscopy. Analyst 2010, 135, 3087-3093.

33. Vargis, E.; Tang, Y.-W.; Khabele, D.; Mahadevan-Jansen, A. Near-infrared Raman Microspectroscopy Detects High-risk Human Papillomaviruses. Transl. Oncol. 2012, 5, 172-179.

34. Rubina, S.; Amita, M.; Kedar, K.D.; Bharat, R.; Krishna, C.M. Raman spectroscopic study on classification of cervical cell specimens. Vib. Spectrosc. 2013, 68, 115-121.

35. Bonnier, F.; Traynor, D.; Kearney, P.; Clarke, C.; Knief, P.; Martin, C.; O’Leary, J.J.; Byrne, H.J.; Lyng, F. Processing ThinPrep cervical cytological samples for Raman spectroscopic analysis. Anal. Methods 2014, 6, 7831-7841.

36. Jemal, A.; Siegel, R.; Ward, E.; Hao, Y.; Xu, J.; Thun, M.J. Cancer statistics, 2009. CA Cancer J. Clin. 2009, 59, 225-249.

37. Howlader, N.; Noone, A.M.; Krapcho, M.; Neyman, N.; Aminou, R.; Waldron, W.; Altekruse, S.F.; Kosary, C.L.; Ruhl, J.; Tatalovich, Z.; et al. SEER Cancer Statistics Review, 1975-2008, 2011. The SEER Web site. Available online: http://seer.cancer.gov/csr/1975_2008/ (accessed on 24 November 2014).

38. Rennemo, E.; Zatterstrom, U.; Boysen, M. Impact of second primary tumors on survival in head and neck cancer: An analysis of 2063 cases. Laryngoscope 2008, 118, 1350-1356. 
39. Hashibe, M.; Brennan, P.; Chuang, S.C.; Boccia, S.; Castellsague, X.; Chen, C.; Curado, M.P.; Dal Maso, L.; Daudt, A.W.; Fabianova, E.; et al. Interaction between tobacco and alcohol use and the risk of head and neck cancer: Pooled analysis in the International Head and Neck Cancer Epidemiology Consortium. Cancer Epidemiol. Biomark. Prev. 2009, 18, 541-550.

40. Hennessey, P.T.; Westra, W.H.; Califano, J.A. Human papillomavirus and head and neck squamous cell carcinoma: Recent evidence and clinical implications. J. Dent. Res. 2009, 88, 300-306.

41. Rethman, M.P.; Carpenter, W.; Cohen, E.E.; Epstein, J.; Evans, C.A.; Flaitz, C.M.; Graham, F.J.; Hujoel, P.P.; Kalmar, J.R.; Koch, W.M.; et al. Evidence-based clinical recommendations regarding screening for oral squamous cell carcinomas. J. Am. Dent. Assoc. 2010, 141, 509-520.

42. Lingen, M.W.; Kalmar, J.R.; Karrison, T.; Speight, P.M. Critical evaluation of diagnostic aids for the detection of oral cancer. Oral. Oncol. 2008, 44, 10-22.

43. Holmstrup, P.; Vedtofte, P.; Reibel, J.; Stoltze, K. Long-term treatment outcome of oral premalignant lesions. Oral. Oncol. 2006, 42, 461-474.

44. Krishna, C.M.; Sockalingum, G.D.; Kurien, J.; Rao, L.; Venteo, L.; Pluot, M.; Manfait, M.; Kartha, V.B. Micro-Raman spectroscopy for optical pathology of oral squamous cell carcinoma. Appl. Spectrosc. 2004, 58, 1128-1135.

45. Cals, F.L.J.; Bakker Schut, T.C.; Koljenović, S.; Puppels, G.J.; de Jong, R.J.B. Method development: Raman spectroscopy-based histopathology of oral mucosa. J. Raman Spectrosc. 2013, 44, 963-972.

46. Malini, R.; Venkatakrishna, K.; Kurien, J.; Pai, K.M.; Rao, L.; Kartha, V.B.; Krishna, C.M. Discrimination of normal, inflammatory, premalignant, and malignant oral tissue: A Raman spectroscopy study. Biopolymers 2006, 81, 179-193.

47. Papamarkakis, K.; Bird, B.; Schubert, J.M.; Miljković, M.; Wein, R.; Bedrossian, K.; Laver, N.; Diem, M. Cytopathology by optical methods: Spectral cytopathology of the oral mucosa. Lab. Invest. 2010, 90, 589-598.

48. Miljkovic, M.; Bird, B.; Lenau, K.; Mazur, A.I.; Diem, M. Spectral cytopathology: New aspects of data collection, manipulation and confounding effects. Analyst 2013, 138, 3975-3982.

49. Byrne, H.J.; Sockalingum, G.D.; Stone, N. Raman Microscopy: Complement or Competitor? In Biomedical Applications of Synchrotron Infrared Microspectroscopy: A Practical Approach; Moss, D., Ed.; RSC Analytical Spectroscopy Monographs, Royal Society of Chemistry: Cambridge, UK, 2011; No. 11.

50. Keating, M.E.; Byrne, H.J. Raman spectroscopy in nanomedicine: Current status and future perspectives. NanoMedicine 2013, 8, 1335-1351.

51. Byrne, H.J.; Baranska, M.; Puppels, G.J.; Stone, N.; Wood, B.; Gough, K.M.; Lasch, P.; Heraud, P.; Sulé-Suso, J.; Sockalingum, G.D. Spectropathology for the Next Generation: Quo Vadis? Analyst 2015, doi:10.1039/C4AN02036G.

(C) 2015 by the authors; licensee MDPI, Basel, Switzerland. This article is an open access article distributed under the terms and conditions of the Creative Commons Attribution license (http://creativecommons.org/licenses/by/4.0/). 